\title{
VARIABLE THICKNESS DIAPHRAGM FOR A STRESS INSENSITIVE WIDEBAND PIEZOELECTRIC MICROMACHINED ULTRASONIC TRANSDUCER
}

\author{
Ofer Rozen ${ }^{1 *}$, Stefon E. Shelton ${ }^{1}$, André Guedes ${ }^{2}$, and David A. Horsley ${ }^{1}$ \\ ${ }^{1}$ University of California Davis, USA \\ ${ }^{2}$ Chirp Microsystems, Inc., USA
}

\section{ABSTRACT}

A novel design for robust, air-coupled piezoelectric micromachined ultrasonic transducers (PMUTs) is presented. The design achieves a 10 -fold reduction in the variation in fundamental frequency created by across-wafer residual stress gradients in the piezoelectric AlN layer. We realized this improvement by designing a thick-layered PMUT diaphragm with an $8 \mu \mathrm{m}$ thick passive layer and a $375 \mu \mathrm{m}$ radius. Simultaneously, we maintained the PMUT's wide bandwidth, required to achieve sufficient axial resolution in range measurement applications, by patterning wedge-shaped ribs that reduce the diaphragm's mass while enhancing stiffness. Compared to a traditional flat plate design with the same initial thickness, surface area, and center frequency, the ribbed designs are predicted to have twice the bandwidth and twice the sound pressure level - bandwidth product (SPL·BW).

\section{INTRODUCTION}

Despite the intensive study of micromachined ultrasonic transducers (MUTs) during the last 20 years, most designs adhere to clamped square and circular plates. The few attempts to modify this convention had different goals than this work. References [13] compared several configurations, targeted to achieve piston-like movement of a capacitive MUT (CMUT), in order to increase the output pressure and the device's active area. However, piezoelectric actuated transducers require a curvature mode shape in order to couple the electrical and the mechanical energy efficiently. For example, a prior study of a circular flexurallysuspended piezoelectric MUT (PMUT) [4] with a a piston-like mode shape, reveals an increased linear operating range at the cost of reducing the electromechanical coupling. Here, a reducedmass/enhanced-stiffness design is demonstrated to improve the transducers' robustness to residual stress, increase their output pressure, and maintain a wide bandwidth.

Air coupled PMUT's can be used for range finding and gesture recognition applications [5-7]. Such applications require an array of identical PMUTs operating at a center frequency from 40$400 \mathrm{kHz}$, a fractional bandwidth greater than $5 \%$, and center frequency variation between PMUTs within the same array that does not exceed the fractional bandwidth [6].

A micromachined ultrasonic transducer's dynamic response, namely its center frequency $(\omega)$ and bandwidth $(\Delta \omega)$, is mainly determined by its mechanical resonance. In a constant-thickness diaphragm, these two parameters are dependent variables. If the diaphragm mechanics is modeled as a lumped second-order massspring-damper $(m, k, b)$ system, they can be expressed as

$$
\begin{aligned}
& \omega=\sqrt{k / m}, \\
& \Delta \omega=b / m
\end{aligned}
$$

where the time-varying displacement of the diaphragm's center is its degree of freedom (DOF).

For a flat, constant-thickness plate, a reference design that we denote as design 0 , the mass $m_{0}$, stiffness $k_{0}$ and damping $b_{0}$ are a functions of the plate surface area $A_{0}$ and thickness $t_{0}$ :

$$
\begin{gathered}
m_{0} \propto A_{0} t_{0} \\
b_{0} \propto A_{0}
\end{gathered}
$$

$$
k_{0} \propto \frac{t_{0}^{3}}{A_{0}}
$$

Therefore, the frequency $\omega_{0}$ and bandwidth $\Delta \omega_{0}$ are:

$$
\begin{gathered}
\omega_{0} \propto \frac{t_{0}}{A_{0}}=\frac{t_{0}}{\pi a^{2}} \\
\Delta \omega_{0} \propto \frac{1}{t_{0}}
\end{gathered}
$$

where $a$ denotes the diaphragm's radius. Residual stress in the thin film layers is known to result in variations in the effective stiffness of the diaphragm, which in turn results in changes in the frequency $\omega_{0}$. A laminated diaphragm with a stressed AlN layer, will have a center frequency $\omega_{0, \text { res }}$ that is shifted relative to that of an unstressed diaphragm, $\omega_{0}$, [8]

$$
\left(\frac{\omega_{0, r e s}}{\omega_{0}}\right)^{2}=\left[1+\frac{a^{2} \sigma t_{0}}{\lambda_{01}^{2} D}\right]
$$

where $\sigma$ is the average stress, $D$ is the flexural rigidity, and $\lambda_{01}$ is a constant defined by the vibration mode-shape of the diaphragm. Because $D \propto t_{0}^{3}$, (4) shows that the stress sensitivity of the center frequency, $S_{\sigma} \propto\left(a / t_{0}\right)^{2}$. Therefore, when comparing PMUTs with constant $\omega_{0}$, the stress sensitivity scales inversely with thickness $t_{0}$. It is therefore possible to reduce the stress sensitivity by increasing the thickness. However, (3) shows that this approach has the undesirable effect of reducing the bandwidth $\Delta \omega_{0}$ by the same factor.

In order to separate the two variables we suggest patterning the diaphragm by selectively reducing its thickness. We denote the novel configuration as design 1 , so its parameters mass $m_{1}$, stiffness $k_{1}$ and damping $b_{1}$ are functions of the new total diaphragm area $A_{1}$ and it maximum thickness $t_{1}$ :

$$
\begin{aligned}
m_{1} & \propto(1-X) A_{1} t_{1} \\
b_{1} & \propto A_{1} \\
k_{1} & \propto \frac{t_{1}^{3}}{A_{1}}(1-Y)
\end{aligned}
$$

where $X$ represents the mass reduction relative to the mass of a constant thickness plate with the same planar geometry, and $Y$ represents the stiffness reduction relative to that of a plate with the same planar geometry.

Assuming that design 1 targets the same frequency as design 0 , $\omega_{1}=\omega_{0}$, the bandwidth can be adjusted by the mass reduction and thickness:

$$
\Delta \omega_{1}=\frac{1}{t_{1}(1-X)}
$$

while the size of the new design is determined by both the change in stiffness and mass:

$$
\frac{A_{0}}{t_{0}}=\frac{A_{1} \sqrt{(1-X)}}{t_{1} \sqrt{(1-Y)}}
$$

As demonstrated by Equations (6-7), introducing $X$ and $Y$ adds two new design variables that broaden the design space.

To verify this approach, we fabricated patterned PMUTs using aluminum nitride (AlN) as the piezoelectric layer. We patterned wedge-shaped ribs that reduce the diaphragm's mass while enhancing its stiffness, in order to realize a design that is both robust to residual stress and has a wide bandwidth. 


\section{DESIGN}

We compare two flat PMUT designs (a thin design having a total thickness of $2 \mu \mathrm{m}$ [8] and a thick design having a total thickness of $9 \mu \mathrm{m}$ ) to five patterned designs. Each patterned design has 8 radial ribs with $0,5,20,35$, and 45 degree rib angle, as shown in Figure 1. The ribbed and the flat thick PMUTs have a $1 \mu \mathrm{m}$ AlN layer and $8 \mu \mathrm{m}$ low-stress $\mathrm{SiO}_{2}$ passive layer, while the thin flat PMUTs are composed of two $1 \mu \mathrm{m}$ AlN layers. Each design has a nominal center frequency of approximately $200 \mathrm{kHz}$.

Table 1: Diaphragm design table

\begin{tabular}{c|cc} 
& Total thickness & Diameter \\
\hline Ref [8] & $2 \mu \mathrm{m}$ & $400 \mu \mathrm{m}$ \\
Flat & $9 \mu \mathrm{m}$ & $770 \mu \mathrm{m}$ \\
Ribs & $9 \mu \mathrm{m}$ & $750 \mu \mathrm{m}$
\end{tabular}

(a)
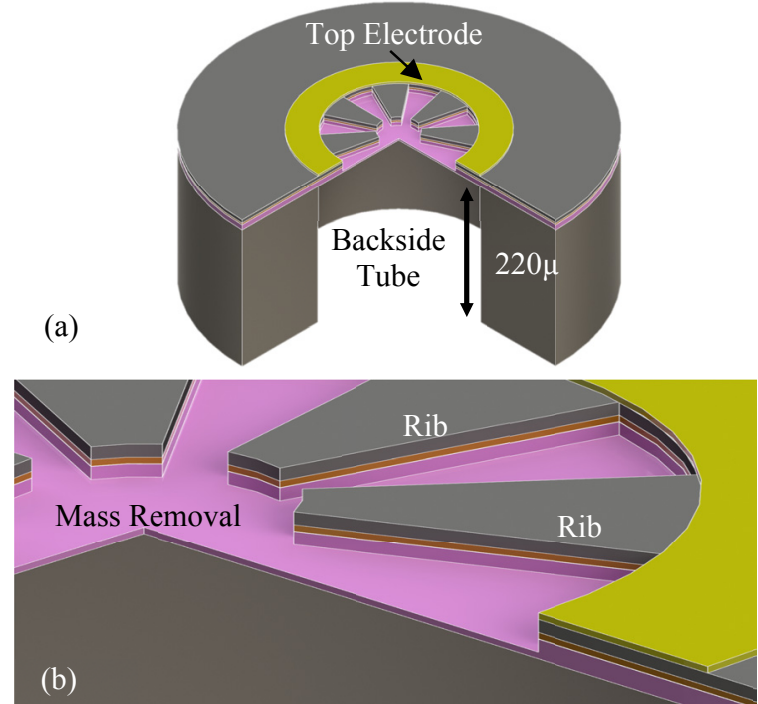

Figure 1: PMUT structure. (a) Reduced-mass/enhanced-stiffness configuration formed by etching a pattern of stiffening ribs into an AlN/SiO ${ }_{2}$ unimorph diaphragm. The etched pattern removes mass from the diaphragm's center. (b) Zoom in on the rib structure.

The different PMUT configurations are spread across the wafer in a few repeating die layouts, depicted in Figure 2. Each die layout was duplicated at different positions on the wafer to allow determination of the across-wafer variation in transducer characteristics.
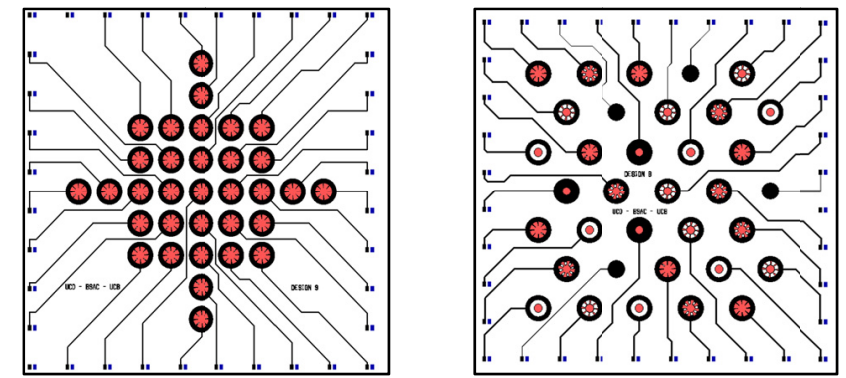

Figure 2: PMUT die layout. (a) 'Cross' die comprised of 36 identical elements. Both flat and $5^{\circ}$ ribs cross dice were fabricated (b) 'Test' die comprised of 35 PMUTs of all different configurations. Both dice were duplicated at different positions on the wafer.

The stress sensitivity of the center frequency and the fractional bandwidth were simulated by finite element method (FEM). Stress sensitivity is found using a nonlinear eigenfrequency model at various residual stress levels. The center frequency and the fractional bandwidth were solved by a three dimensional axisymmetric piezo-acoustic model implemented in COMSOL.

As shown in Figure 3, it is possible to reduce the effect of stress on center frequency by increasing the thickness of the flat plate PMUT; however, this approach has the undesirable effect of reducing the bandwidth. The rib pattern, in contrast, reduces stress sensitivity without significantly reducing the bandwidth. The FEM indicates that the thick design reduces the sensitivity of the resonance frequency to residual stress by a factor of 10 compared to thin diaphragms with the same fundamental frequency. The simulations also indicate that the ribbed designs are expected to have twice the bandwidth and twice the sound pressure level bandwidth product (SPL-BW) in comparison to the thick PMUT design.

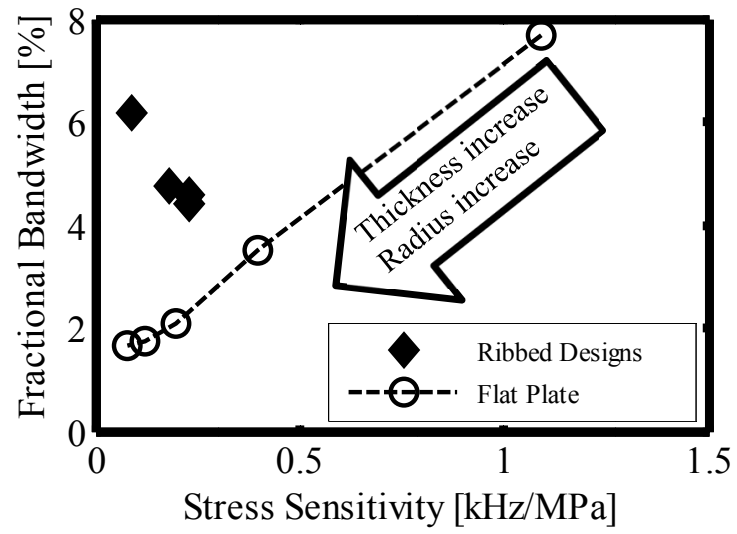

Figure 3: Stress sensitivity versus fractional bandwidth for $200 \mathrm{kHz}$ PMUTs, found by FEM. The diamonds show five ribbed PMUT designs, with rib angle varying from $0^{\circ}-45^{\circ}$, that have low stress sensitivity while also maintaining wide bandwidth.

\section{FABRICATION}

The process starts with a $150 \mathrm{~mm}$ silicon wafer with an $8 \mu \mathrm{m}$ silicon dioxide layer on top. Then, Mo $(150 \mathrm{~nm}) / \mathrm{AlN}(1 \mu \mathrm{m})$ layers are deposited as the bottom electrode and active layer respectively. Then an $\mathrm{Al}$ top electrode is deposited and patterned and the bottom electrode via is opened by etching through the AIN. Next, the AIN, the bottom electrode and the $\mathrm{SiO}_{2}$ structural layer are patterned using reactive-ion etching (RIE) into $3.5 \mu \mathrm{m}$ deep ribs. Finally, the wafer is thinned to $220 \mu \mathrm{m}$ by grinding and the membrane is released by etching the backside Si using deep reactive-ion etching (DRIE).

(a)

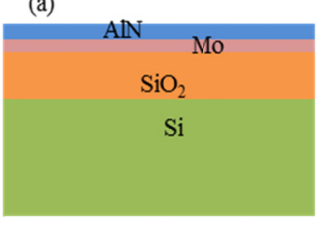

(b)

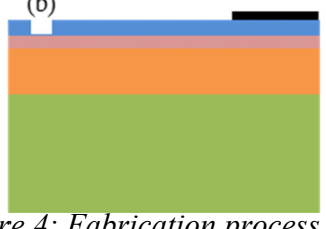

Figure 4: Fabrication process. (c)

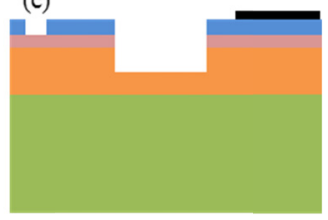

(d)

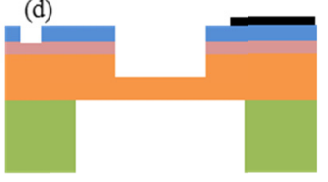




\section{RESULTS}

Wafer-level measurements of PMUT impedance versus frequency were collected using a semi-automated probe station. A representative frequency response is shown in Figure 5. The impedance data were fit with a Butterworth Van Dyke (BVD) model, allowing extraction of the motional impedance components $\left(\mathrm{R}_{\mathrm{x}}, \mathrm{L}_{\mathrm{x}}, \mathrm{C}_{\mathrm{x}}\right)$ and electrical impedance components $\left(\mathrm{R}_{\mathrm{s}}, \mathrm{C}_{0}\right)$ as well as center frequency $(2 \pi f=\omega)$ and quality factor $(Q)$, Figure 5 . All the mean and standard deviation values reported are the robust values, found using Huber's M-estimation in JMP ${ }^{\circledR}$ 11.0.
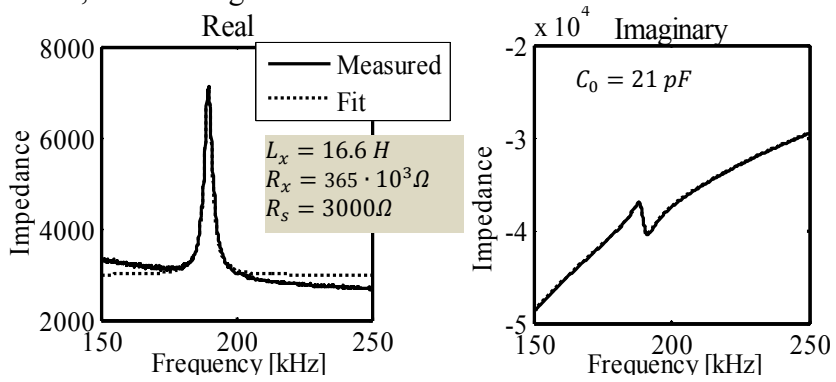

Figure 5: A typical frequency response. From the fit of the real part (left) the electric resistance and the mechanical serial equivalent $R_{x}-L_{x}-C_{x}$ are found and transformed to the mechanical domain into the acoustic damping, effective mass and the stiffness of the diaphragm. From the imaginary part (right), the transducer capacitance $C_{0}$ is found.

The center frequency of a total 694 devices of different configurations is analyzed. For both flat and $5^{\circ}$ ribs configurations, the histograms, Figure 6, show the center frequency distribution of all such devices. The variation in frequency for the ribbed configuration is half of that of the flat configuration.

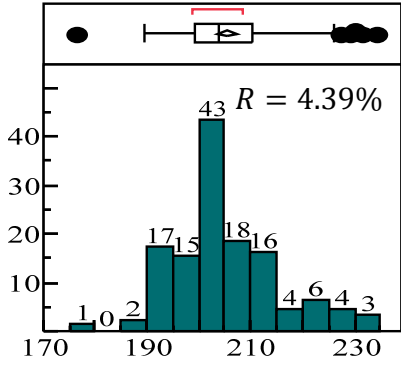

(a) Center Frequency $[\mathrm{kHz}]$

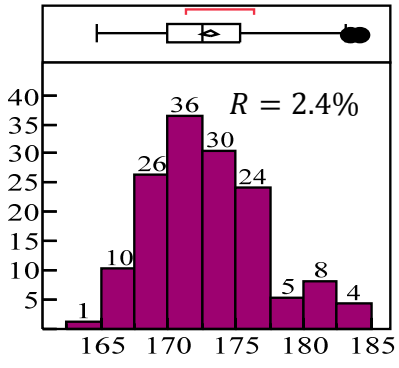

(b)
Figure 6: Center frequency histogram for (a) flat and (b) $5^{\circ}$ ribs configurations measured at all points across the wafer. $R$ is the robust standard deviation divided by the robust mean.

Results for all configurations were analyzed using the test dice and are shown in Figure 7. While the measured center frequency is higher the simulation's prediction, the differences between the configurations match well with the FEM results.

The simulations also indicated that the ribbed design's bandwidth would be twice that of the flat design. However, the wafer-level measurements indicate little difference in bandwidth for the flat and ribbed designs and both designs have a much higher $Q$ than anticipated. This is due to the fact that the tube beneath each PMUT is not open to the air and is instead closed by the wafer chuck in probe-station measurements.
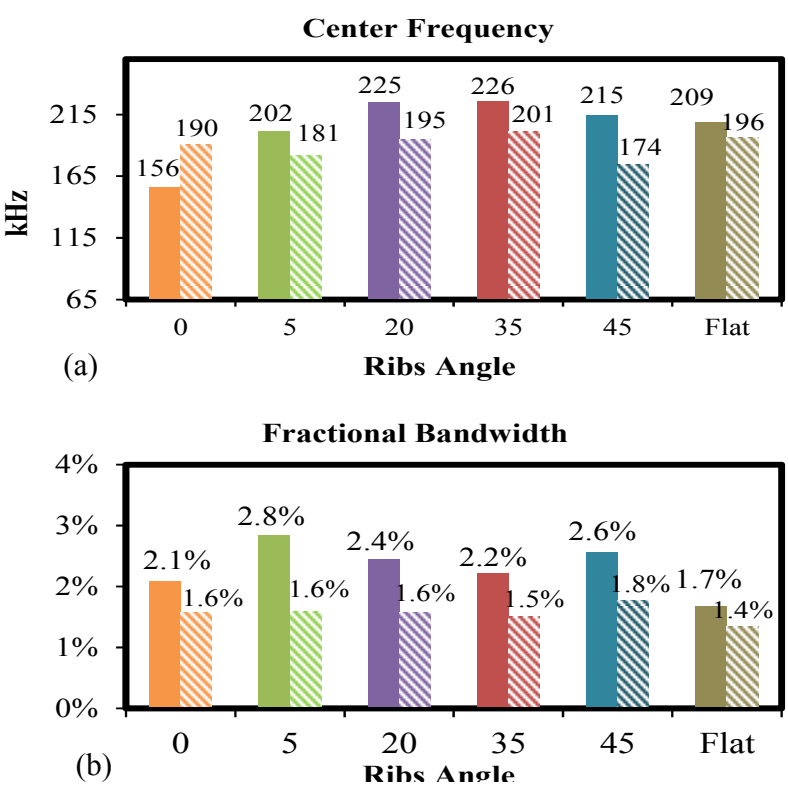

Figure 7: simulated (solid) and measured (hatched) (a) center frequency and (b) fractional bandwidth measured with a closed backside tube. (Simulated with an open backside tube)

Across-Die and Across-Wafer Matching of Center Frequency

A key performance metric is the variation of the PMUT center frequency from the average center frequency across each die and across each wafer. This variation is attributed to differences in PMUT geometry (diameter and film thickness) and residual stress. A wafer map of the mean and standard deviation of center frequency for the two cross dice (Fig. 2a) is shown in Figure 8.

The frequency variation of each design relative to the average frequency measured across the wafer $(\Delta f / \bar{f})$ is summarized in Table 2. While previous work [6] showed an across wafer variation of about $70 \%$, the maximum variation measured in this work is only $8.1 \%$, and the average variation of each design is $2.4 \%-4.9 \%$.
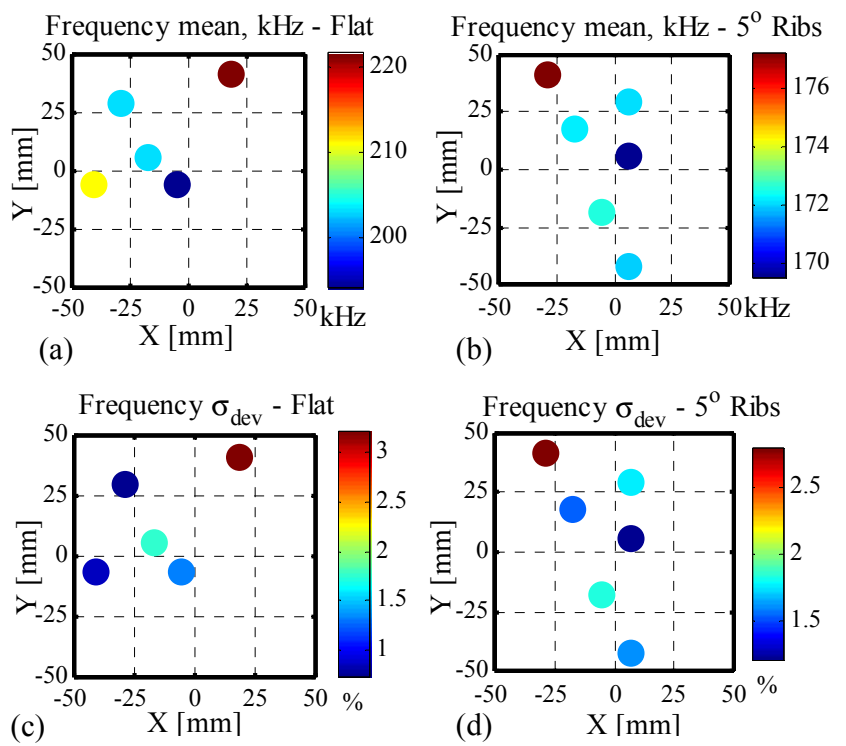

Figure 8: (a-b) Dice's robust mean center frequency and (c-d) robust standard deviation normalized by the mean center frequency, for flat and $5^{\circ}$ ribs configuration. $X$ and $Y$ indicate the dice center position, relative to the mask center. 
Table 2: Deviation of center frequency relative to wafer average.

\begin{tabular}{c|cccc} 
Config. & Minimum & Maximum & Average & $\boldsymbol{N}$ \\
\hline $\boldsymbol{0}^{\circ}$ & $3.1 \%$ & $5.8 \%$ & $4.2 \%$ & 3 \\
$\mathbf{5}^{\circ}$ & $2.9 \%$ & $4.8 \%$ & $3.8 \%$ & 4 \\
$\mathbf{2 0}^{\circ}$ & $3.3 \%$ & $4.7 \%$ & $4.1 \%$ & 3 \\
$\mathbf{3 5}^{\circ}$ & $2.8 \%$ & $6.2 \%$ & $4.0 \%$ & 3 \\
$\mathbf{4 5}^{\circ}$ & $0.6 \%$ & $12 \%$ & $2.4 \%$ & 35 \\
Flat & $1.3 \%$ & $8.1 \%$ & $4.9 \%$ & 35
\end{tabular}

The across-die variation of center frequency was analyzed for cross shaped arrays, each containing 36 PMUTs, as shown in Figure 2a. From Figure 9 it is clear that the die layout influences the frequency variation. PMUTs on the edge of the cross array are etched faster, have bigger radius and therefore lower frequency. This variation can be eliminated in future layouts by patterning dummy PMUTs around the array in order to maintain similar boundary conditions in the final DRIE step.
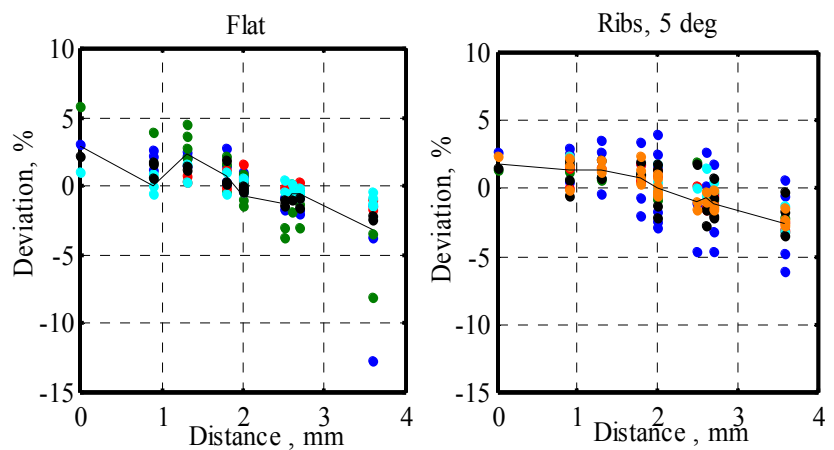

Figure 9: Center frequency deviation as a percentage of the die's mean frequency $v$ s. distance of the PMUT from the center of the die. Different colors represent different dice. The solid line indicates the mean value at each location.

The across die variation robust statistics are shown in

Table 3. We are achieving low and robust variation, as the results are consistent across the wafer, improving the future manufacturing yield.

Table 3: Deviation of center frequency relative to die average

\begin{tabular}{c|cccc} 
Config. & Minimum & Maximum & Average & N \\
\hline $\mathbf{5}^{\circ}$ & $0.7 \%$ & $3.2 \%$ & $1.6 \%$ & 5 \\
Flat & $1.2 \%$ & $2.8 \%$ & $1.8 \%$ & 6
\end{tabular}

\section{AIN Material Properties}

The PMUT system can be modeled using a lumped parameter model [5]. Using the fitted model, the piezoelectric coefficient $e_{31}$, of the AlN and its dielectric constant $\varepsilon_{11}$ can be extracted, Table 4, assuming a known geometry, density, Young modulus and Poisson ratio of all the layers.

Table 4: AlN material properties

\begin{tabular}{c|cc} 
& Literature [9] & This work \\
\hline $\boldsymbol{\varepsilon}_{\mathbf{1 1}}$ & 9.5 & 8.45 \\
$\boldsymbol{e}_{\mathbf{3 1}}$ & -0.58 & -0.55
\end{tabular}

\section{CONCLUSIONS}

We present a variable thickness diaphragm design that simultaneously improves several performance metrics. Six different configurations were compared, with center frequencies ranging between $190 \mathrm{kHz}$ to $209 \mathrm{kHz}$. The across wafer variation in the center frequency of the ribbed designs is $4 \%$, and their across die variation is $1.6 \%, 10$ times better than previously fabricated flat thin configurations. Future work includes acoustic measurements of the pressure output as well as the frequency response in completely open backside conditions to verify the predicted bandwidth improvement of the rib design.

\section{ACKNOWLEDGMENTS}

The authors thank Ira Wygant, Wei Yan Shih, and Philippe Jansen at Texas Instruments for assistance with wafer-level test and data analysis. Devices were fabricated in the UC Berkeley Marvell Nanofabrication Laboratory.

Travel support has been generously provided by the Transducer Research Foundation.

\section{REFERENCES}

[1] Y. Huang, X. Zhuang, E. Hggstrom, A. S. Ergun, C. H. Cheng, and B. T. Khuri-Yakub, "Capacitive micromachined ultrasonic transducers with piston-shaped membranes: Fabrication and experimental characterization," in Trans. Ultrason., Ferroelect., Freq. Contr., vol. 56, pp. 136-145, 2009.

[2] A. Nikoozadeh and P. T. Khuri-Yakub, "CMUT with substrate-embedded springs for non-flexural plate movement," in IEEE Ultrason. Symp, 2010, pp. 1510-1513.

[3] B. C. Lee, A. Nikoozadeh, K. K. Park, and B. T. KhuriYakub, "Understanding CMUTs with Substrate-Embedded Springs," in IEEE Ultrason. Symp. , 2011, pp. 1008-1011.

[4] A. Guedes, S. Shelton, R. Przybyla, I. Izyumin, B. Boser, and D. A. Horsley, "Aluminum nitride pMUT based on a flexurally-suspended membrane," in Transducers, 2011, pp. 2062-2065.

[5] R. J. Przybyla, S. E. Shelton, A. Guedes, Izyumin, II, M. H. Kline, D. A. Horsley, et al., "In-Air Rangefinding With an AlN Piezoelectric Micromachined Ultrasound Transducer," IEEE Sensors J., vol. 11, pp. 2690-2697, Nov 2011.

[6] S. Shelton, A. Guedes, R. Przybyla, R. Krigel, B. Boser, and D. Horsley, "Aluminum Nitride Piezoelectric Micromachined Ultrasound Transducer Arrays," in Hilton Head, 2012, pp. 291-294.

[7] R. J. Przybyla, S. E. Shelton, A. Guedes, R. Krigel, D. A. Horsley, and B. E. Boser, "In-Air Ultrasonic Rangefinding and Angle Estimation Using an Array of AlN Micromachined Transducers," in Hilton Head, 2012, vol. 7, p. 3.

[8] S. Shelton, C. Mei-Lin, P. Hyunkyu, D. Horsley, B. Boser, I. Izyumin, et al., "CMOS-compatible AlN Piezoelectric Micromachined Ultrasonic Transducers," in IEEE Ultrason. Symp., 2009, pp. 402-405.

[9] A. V. Sotnikov, H. Schmidt, M. Weihnacht, E. P. Smirnova T. Y. Chemekova, and Y. N. Makarov, "Elastic and piezoelectric properties of AlN and LiAlO2 single crystals," in Trans. Ultrason., Ferroelect., Freq. Contr. vol. 57, pp. 808$811,2010$.

\section{CONTACT}

*O. Rozen, tel: +1-530-219-8461; orozen@ucdavis.edu 